\title{
Normal Distribution
}

National Cancer Institute

\section{Source}

National Cancer Institute. Normal Distribution. NCI Thesaurus. Code C53215.

A family of probability density functions whose mean, median and mode are identical.

The function generates a symmetrical curve, whose position and shape is determined by its location and scale parameters, the mean and standard deviation respectively. The standard normal distribution has a location parameter of 0 and a scale parameter of 1 . 